\title{
Physical activity and depression in adolescents: cross-sectional findings from the ALSPAC cohort
}

\author{
Nicola J. Wiles $\cdot$ Anne M. Haase $\cdot$ Debbie A. Lawlor • \\ Andy Ness · Glyn Lewis
}

Received: 19 April 2011/Accepted: 26 July 2011/Published online: 9 August 2011

(C) The Author(s) 2011. This article is published with open access at Springerlink.com

\begin{abstract}
Purpose Few studies have examined the association between physical activity (PA), measured objectively, and adolescent depressive symptoms. The aim of this study was to determine whether there is an association between objective measures of PA (total PA and time spent in moderate and vigorous PA (MVPA)) and adolescent depressive symptoms.

Methods Data on 2,951 adolescents participating in ALSPAC were used. Depressive symptoms were measured using the self-report Mood and Feelings Questionnaire (MFQ) (short version). Measures of PA were based on accelerometry. The association between PA and MFQ scores was modelled using ordinal regression.

Results Adolescents who were more physically active (total PA or minutes of MVPA) had a reduced odds of
\end{abstract}

Electronic supplementary material The online version of this article (doi:10.1007/s00127-011-0422-4) contains supplementary material, which is available to authorized users.

N. J. Wiles $(\square) \cdot$ G. Lewis

Academic Unit of Psychiatry, School of Social and Community

Medicine, University of Bristol, Oakfield House,

Oakfield Grove, Bristol BS8 2BN, UK

e-mail: nicola.wiles@bristol.ac.uk

\section{A. M. Haase}

Centre for Exercise, Nutrition and Health, School of Policy

Studies, University of Bristol, Bristol, UK

D. A. Lawlor

MRC Centre for Causal Analyses in Translational

Epidemiology, School of Social and Community Medicine,

University of Bristol, Bristol, UK

A. Ness

School of Oral and Dental Science, University of Bristol,

Bristol, UK depressive symptoms [OR ${ }_{\text {adj }}$ total PA (tertiles): medium 0.82 (95\% CI: 0.69, 0.97); high 0.69 (95\% CI: 0.57, 0.83)]; $\mathrm{OR}_{\text {adj }}$ per 15 min MVPA: 0.92 (95\% CI: 0.86, 0.98). In a multivariable model including both total PA and the percentage of time spent in MVPA, total PA was associated with depressive symptoms $\left(\mathrm{OR}_{\mathrm{adj}}\right.$ total PA (tertiles): medium 0.82 (95\% CI: 0.70, 0.98); high 0.70 (95\% CI: $0.58,0.85$ ) but the percentage of time spent in MVPA was not independently associated with depressive symptoms $\left[\mathrm{OR}_{\text {adj }}\right.$ MVPA (tertiles) medium 1.05 (95\% CI: 0.88, 1.24), high 0.91 (95\% CI: 0.77, 1.09)].

Conclusions The total amount of PA undertaken was associated with adolescent depressive symptoms, but the amount of time spent in MVPA, once total PA was accounted for, was not. If confirmed in longitudinal studies and randomised controlled trials, this would have important implications for public health messages.

Keywords Physical activity - Depressive symptoms . Adolescence - ALSPAC

\section{Introduction}

Depression is ranked amongst the top five contributors to the global burden of disease [28], and its prevalence is increasing amongst young people [25]. Adolescence is a critical period and depression during this time may impact on educational attainment, and is also associated with an increased likelihood of recurrence of symptoms later in life [27]. Potentially modifiable risk factors, such as physical activity (PA), are therefore of great interest.

Promotion of PA is at the top of the government's public health agenda [10], and current recommendations state that children should accumulate (at least) $1 \mathrm{~h}$ of moderate to 
vigorous physical activity (MVPA) every day [6, 10, 54]. However, there is little evidence for health benefits of such levels of PA [46, 59], particularly in terms of the effects on mental health [59].

Amongst children, most of the evidence on PA and mental health is based on cross-sectional studies [1, 3, 14, 17, 19, 24, 32, 39, 41, 44, 53, 56, 57, 60]. Most support an association between more frequent PA and lower levels of depression amongst adolescents $[3,14,17,19,24,32,39$, $41,44,53,57,60]$, although there are exceptions [1, 26, 56]. In many, the association between PA and depressive symptoms is modest $(r=-0.14$ to -0.18$)[32,39,41,44$, 57], and it is possible that confounding may account for these associations. Few studies have adjusted for confounders, with those that did mostly limiting this to age, gender and socioeconomic background [1, 3, 53, 56], and such adjustment often attenuated the association [1, 19]. Few have adjusted for adolescent substance use [19] or obesity [3, 19], and only one longitudinal study has adjusted for the timing of puberty [55]. These three factors may be important confounders of an association between PA and adolescent mood.

The main methodological weakness of prior studies has been the reliance on subjective measures of PA. In addition, measurement of PA should incorporate the frequency, duration and intensity of each activity [23]. Whilst most prior studies record frequency of activities, just over half make reference to the intensity $[1,15,32,39,41,45,53$, $56]$ and/or duration $[1,15,17,19,39,41,45,56]$ of these activities. None of these studies examined the importance of the intensity of the activity in terms of mental health benefits. Thus, whilst recommendations for PA are framed in terms of time spent in MVPA, there is little robust empirical evidence to support this for mental health outcomes.

In the literature, there is much discussion about the measurement of PA in children and comparisons of the relative merits of subjective versus objective measures $[8$, $18,51,62]$. The gold standard for measuring total energy expenditure (the doubly labelled water method) is too costly for widespread use [62] and, importantly, cannot be used to estimate PA undertaken at different levels of intensity [51]. Self-report questionnaires are cheap to administer and thus are intuitively attractive for use in large-scale studies. However, concerns have been voiced about the ability of children to accurately recall PA [4, 18, 49]. As such, these measures may be prone to substantial measurement error that may obscure or reduce associations.

In light of this, objective measures of PA are increasingly being used [58]. Such measures are not without problems [18], but they will more accurately reflect habitual PA. A recent systematic review highlighted the paucity of evidence regarding objective measurements of
PA and mental health in children and adolescents [20]. This review also highlighted the need for future studies to examine the effect of different intensities of activity on different dimensions of health [20]. To date, to the best of our knowledge, only three studies have examined the association between an objective measure of PA and depressive symptoms. One of these used pedometers, which do not capture the intensity of the PA [42]. Two others have measured PA using accelerometry [21, 43]. Secondary analysis of baseline data from the Trial of Activity for Adolescent Girls (TAGG) found no evidence of an association between PA and depressive symptoms [21]. Another study of 9- to 10-year-old primary school children found weak evidence to support an association between vigorous PA assessed by accelerometry and depressive symptoms $(r=-0.18)$, with the inverse noted for very light activities, such that undertaking more light activities was associated with an increased likelihood of depressive symptoms $(r=0.35)$ [43]. Whilst focussing on the intensity of activity, this study was small $(n=57)$ and only adjusted for the potential confounding effect of body fat.

Therefore, we aimed to investigate the association between physical activity (in terms of both total PA and time spent in MVPA), measured objectively, and depressive symptoms in a large UK population-based cohort (the Avon Longitudinal Study of Parents and Children, ALSPAC) using cross-sectional data available at age 14 years.

\section{Methods}

\section{The Avon Longitudinal Study of Parents and Children}

Full details of the ALSPAC study (www.bristol.ac.uk/ alspac) have been published elsewhere [16]. Briefly, all women residents in the former region of Avon, UK, with an expected delivery date between 1st April 1991 and 31st December 1992 were eligible to take part in ALSPAC and were enrolled in early pregnancy. Ethical approval for the study was obtained from the ALSPAC Ethics and Law Committee and the local research ethics committees. Within ALSPAC, 12,942 singleton infants were born at term ( $\geq 37$ completed weeks).

\section{Outcome}

The primary outcome was self-report of depressive symptoms as measured by the short version of the Mood and Feelings Questionnaire (MFQ) [2, 34] (http://devepi.mc. duke.edu/mfq.html). This is a 13-item checklist of symptoms experienced in the previous 2 weeks. Each item is scored zero (not true), one (sometimes true) or two (true) giving a 
total MFQ score of 0-26. Each adolescent completed the MFQ as part of the clinic assessment at age 14 years [Teen Focus2 clinic: mean age, 13.8 years (SD 0.21)].

Whilst depressive symptoms exist as a continuum in the population, it was not possible to utilise these as continuous data due to the highly positively skewed distribution of scores (even after the data were subjected to various transformations). Alternative distributions such as the Poisson or negative binomial are only appropriate for count data. Therefore, rather than creating a binary outcome variable (depressed vs. not depressed), to maximise the efficiency of our analyses, we defined our primary outcome as an ordinal variable that represented approximate tertiles of the distribution (MFQ score: $0-2 ; 3-5 ; \geq 6$ ).

\section{Exposures}

Full details of the measurement of physical activity in the ALSPAC cohort have been reported previously [29-31]. In brief, all children who attended the clinic at age 14 years were asked to wear an Actigraph AM7164 2.2 accelerometer (Actigraph LLC, Fort Walton Beach, FL, USA) around their waist, at the right hip, for 7 days. The accelerometers were to be worn during waking hours except for when showering, bathing or participating in water sports.

The accelerometers recorded data as counts, which are averaged over a defined period (in ALSPAC, $1 \mathrm{~min}$ ). Physical activity variables were derived from the raw accelerometer counts using customised software. Data from children who had worn the accelerometer for at least $10 \mathrm{~h}$ a day for at least 3 days were considered valid. These criteria have previously been shown to achieve the greatest power and good reliability [31]. Ten or more minutes of consecutive zeros were regarded as periods in which the monitor was unworn, and these were deleted from each file.

One measure of total PA that has been validated against doubly labelled water [13] is the average accelerometer counts per minute (cpm) over the full period of valid recording. However, as this measure reflects both the amount and intensity of PA undertaken, for the present analyses, the average daily minutes of total PA at light, moderate or vigorous intensity was calculated based on cut-points of $200-3,599,3,600-6,199$ and $\geq 6,200 \mathrm{cpm}$, respectively. These cut-points were based on the results of a calibration study where a subsample of 246 ALSPAC participants was asked to perform a series of everyday activities whilst wearing an actigraph and a portable metabolic unit (Cosmed K4bz, Cosmed, http:///www.cosmed.it) [29]. The cut-point for MVPA corresponded to four times resting metabolic rate (equivalent to brisk walking). Examples of MVPA include aerobic activities such as cycling or swimming that increase the heart rate, whilst more strenuous activities such as squash or running would be classified as vigorous activity. Minutes of MVPA was calculated as the average minutes of such activity per valid day of measurement.

\section{Potential confounders}

Data on a large number of potential confounders were available at various time points. These included: (1) gender; (2) age; (3) markers of socioeconomic position (maternal education recorded on the antenatal questionnaire completed at 32 weeks (highest qualification: $<$ 'O' level; ' $O$ ' level; >'O' level, where ' $O$ ' levels are ordinary-level qualifications usually completed at age 16 years, the minimum legal age at which pupils in the UK can leave full-time education); housing tenure recorded on the antenatal questionnaire completed at 8 weeks [owned (with/without mortgage); rented; other]; household overcrowding recorded on the antenatal questionnaire completed at 8 weeks $(<1$ person per room; 1 person per room; $>1$ person per room); access to a car recorded on the antenatal questionnaire completed at 8 weeks (yes/no); highest parental social class recorded on the antenatal questionnaire completed at 32 weeks (based on Registrar General Occupational Classification [40]: I; II; IIINM; IIIM/IV/V) where I (senior managerial/professional) is the highest and $\mathrm{V}$ (manual unskilled) the lowest socioeconomic occupation); (4) obesity [fat mass was derived using a Lunar Prodigy DXA scanner (GE Medical Systems Lunar, Madison, WI, USA)] [47] and height [measured using a Harpenden Stadiometer (Holtain, Crymych, Pembs, UK) at the clinic held at age 14]; (5) substance use recorded at the clinic held at age 14 years (binge drinking defined as ever drinking 3 or more alcoholic drinks in a $24 \mathrm{~h}$ period [11] (yes/no); cigarette smoking (never smoked; has smoked cigarettes, but not in the past 6 months; has smoked cigarettes in the past 6 months); and whether they had ever tried cannabis (yes/no)].

\section{Dataset}

In total, 5,423 children attended the clinic at age 14 years and were asked to wear an accelerometer for the following week. Data on physical activity were available for 3,357 children (62\% of clinic attenders) who had worn the accelerometer for at least $10 \mathrm{~h}$ per day for at least 3 days [31]. The majority of these $(n=3,298)$ had completed the MFQ in the clinic. Data on potential confounders (listed in items 1-5 above) were available for 2,951 adolescents.

\section{Statistical analysis}

All analyses were conducted using Stata version 10.1 [52].

Preliminary analyses found that there was evidence of a non-linear association between average daily minutes of 
total PA and depressive symptoms; therefore, total PA was split by tertiles for further analyses.

Ordinal regression using a proportional odds model was used to examine the relationship between physical activity (total PA (tertiles: $\leq 270 \mathrm{~min} ;>270-326.6 \mathrm{~min}$; $\geq 326.7$ min) and MVPA (per $15 \mathrm{~min}$ )) and depressive symptoms (approximate tertiles of MFQ score: $0-2 ; 3-5$; $\geq 6$ ). Odds ratios (OR) and their $95 \%$ confidence intervals $(95 \% \mathrm{CI})$ are reported. Univariable models were subsequently adjusted for potential confounders in an incremental manner: (1) gender; (2) age; (3) markers of socioeconomic position (SEP); (4) obesity; and (5) substance use. Whilst data on pubertal stage are recorded as part of ALSPAC, no data on pubertal stage were collected at the time of the clinic (on average, such data were collected 8.6 months later). Therefore, to examine the potential confounding effect of puberty, analyses were restricted to girls and further adjusted for the timing of menarche (based primarily on data from a series of six postal questionnaires that focused on pubertal development). All models were adjusted for the time that the accelerometer was worn.

The OR obtained from a proportional odds model is an estimate of the association between the independent variable (the measure of PA) and all levels of the outcome (depressive symptoms) and is essentially a summary of the binary logistic odds ratios for each of the cut-points (0-2 vs. $3-5$ and $\geq 6 ; 0-2$ and $3-5$ vs. $\geq 6$ ). The assumption of homogeneity of effect across cut-points of depressive symptoms was tested formally using the omodel option in Stata. There was little evidence to suggest heterogeneity of effect across cut-points. Thus, the OR can be interpreted as the effect per category of exposure on having more depressive compared to less depressive symptoms. An OR of less than 1.00 indicates that those who are more physically active have fewer depressive symptoms.

Three prior studies have suggested gender differences $[15,35,45]$, but only one study has formally tested for an interaction and found no evidence to support a differential effect [1]. Therefore, within the ALSPAC dataset, an interaction between gender and the measure of PA was introduced into the final regression models and the strength of evidence to support an interaction determined using the Wald test.

To determine the importance of the amount versus the intensity of the physical activity, it was necessary to respecify the measure of MVPA. Including both total PA (defined as average daily total of minutes of activity) and minutes of MVPA in the same regression model means that it would not have been possible to examine the independent effect of increasing MVPA whilst holding total PA constant (as increasing MVPA would, by definition, have increased total PA). Therefore, an additional variable was created that represented the percentage of total time spent in MVPA (minutes of MVPA/total minutes of PA at light, moderate or vigorous intensity $\times 100$ ). Thus, by including both total minutes of activity and percentage of time spent in MVPA in one model, it would be possible to determine the effect of increasing the percentage of time spent in MVPA whilst holding the amount of activity (minutes of total PA) constant. This would then answer the question of whether it is important to increase the total amount of activity that adolescents undertake or the intensity at which they do that activity.

Both total PA (average daily total minutes of activity) and percentage of time spent in MVPA were split by tertiles to define those with low, medium and high levels of these two variables for subsequent analyses. Regression models were then re-run, adjusting for potential confounders as outlined earlier, to first model the association between tertiles of percentage of time spent in MVPA and adolescent mood to enable comparison with the earlier analyses. Regression models were then repeated including both tertiles of total PA (average daily total of minutes of physical activity) and tertiles of percentage of time spent in MVPA $(<4.87 \% ; 4.87-8.95 \%$; $\geq 8.96 \%)$ in the same model to determine the independent effects of these variables and thus address the question of the importance of the intensity versus the amount of PA in relation to adolescent mood.

\section{Missing data}

Sensitivity analyses were conducted to examine the influence of missing data on the findings. Missing data were imputed using the method of multiple imputation by chained equation (MICE) [61] (Stata ice procedure version 1.9.4 dated 24 March 2011). One imputation model was generated for each measure of PA. The imputation model included the measure of physical activity, MFQ score, all potential confounders plus additional markers of socioeconomic status (housing tenure and access to a car when the child was aged 8 months; maternal education recorded when the child was aged 61 months), and childhood adiposity (DXA fat mass) and height recorded at the age 12 clinic (Focus@11).

The additional variables included in the imputation model either represented the first occasion of measurement of a potential confounder, which would provide the most complete information on this variable, or data from subsequent time point(s), where available. Variables were included in the imputation model and declared as continuous, binary, categorical or ordinal variables as appropriate. The match procedure was used to handle non-normally distributed variables, which could not be successfully transformed (DXA fat mass). In total, 25 datasets were generated and 10 switching procedures were used. 


\section{Results}

Description of cohort

In total, 5,423 adolescents attended the ALSPAC clinic at age 14 years, with 3,298 adolescents completing the MFQ during this visit and providing objective data on physical activity (based on accelerometry). Just over half (53\%) were girls and their mean age at the time of the clinic visit was 13.8 years (SD 0.2). Data on potential confounders were available for $89 \%$ of this group $(n=2,951 ; 54 \%$ of clinic attenders).

\section{Depressive symptoms}

The median score on the MFQ at age 14 years was 4 [interquartile range (IQR): 2, 7]. Girls scored higher on the MFQ (median: 4 [IQR: 2, 8] than boys (median: 3 [IQR: 1, 5]) (Kruskal-Wallis: $p<0.001$ ).

Physical activity

Overall, the adolescents undertook, on average, $21 \mathrm{~min}$ of MVPA per day (median: IQR [12, 33]). Those who were more active at moderate/vigorous intensity were more active overall (Spearman's rho for minutes of MVPA vs. total PA $=0.52$ ).

Boys were more active than girls in terms of both total physical activity (average daily minutes at light, moderate or vigorous intensity-boys: median, 319 [IQR: 278, 364]); girls: median 281 [IQR: 247, 322]; Kruskal-Wallis $p<0.001$ ) and minutes of MVPA (boys: median 25 min [IQR: 15, 39]; girls: median 17 min [IQR: 10, 27]: KruskalWallis $p<0.001)$. The percentage of time spent in MVPA was only $6.9 \%$ (median: IQR $[4.1,10.4]$ ). The correlation between percentage of time spent in MVPA and total physical activity was more modest (Spearman's rho $=0.28$ ).

There was some evidence that adolescents of lower socioeconomic position (SEP) (based on indicators such as maternal education and household overcrowding) were more active, but this was not consistent across all such markers (Web Appendix 1). Adolescents who were above average in terms of their total fat mass at age 14 years (as measured by DXA) were less active. In addition, those who binge drank, smoked cigarettes or who had ever tried cannabis were more active in terms of both total PA and time in MVPA (Web Appendix 1).

Total physical activity and self-reported depressive symptoms at age 14 years

In univariable analyses, those adolescents who had higher levels of total PA (in terms of average daily minutes at light, moderate or vigorous intensity) had a reduced odds of depression [OR (tertiles): medium, 0.73 (95\% confidence interval (95\% CI): 0.62, 0.86); high, 0.58 (95\% CI: 0.49, 0.69)] (Table 1). The magnitude of this association attenuated following adjustment for gender (OR (tertiles): medium 0.80 (95\% CI: 0.67, 0.94); high 0.69 (95\% CI: $0.58,0.83)$ ) (Table 1). However, further adjustment for a number of other potential confounders did not substantially alter the association (Table 1).

Table 1 Cross-sectional associations between measures of physical activity and depressive symptoms in 14-year-old adolescents

\begin{tabular}{|c|c|c|c|c|c|c|c|c|}
\hline \multirow[t]{3}{*}{ Outcome } & \multirow[t]{3}{*}{$n$} & \multicolumn{5}{|c|}{$\begin{array}{l}\text { Total PA (tertiles of average daily minutes at light, } \\
\text { moderate or vigorous intensity) }\end{array}$} & \multicolumn{2}{|c|}{$\begin{array}{l}\text { MVPA per } \\
15 \text { min }\end{array}$} \\
\hline & & \multirow{2}{*}{$\begin{array}{l}\text { Low } \\
\text { OR }\end{array}$} & \multicolumn{2}{|c|}{ Medium } & \multicolumn{2}{|l|}{ High } & \multirow[t]{2}{*}{$\mathrm{OR}^{*}$} & \multirow[t]{2}{*}{$95 \% \mathrm{CI}$} \\
\hline & & & $\mathrm{OR}^{*}$ & $95 \% \mathrm{CI}$ & $\mathrm{OR}^{*}$ & $95 \% \mathrm{CI}$ & & \\
\hline Unadjusted & 2,951 & 1.00 & 0.73 & $0.62,0.86$ & 0.58 & $0.49,0.69$ & 0.87 & $0.82,0.93$ \\
\hline \multicolumn{9}{|l|}{ Adjusted for } \\
\hline Gender & 2,951 & 1.00 & 0.80 & $0.67,0.94$ & 0.69 & $0.58,0.83$ & 0.93 & $0.87,0.99$ \\
\hline+ Age & 2,951 & 1.00 & 0.80 & $0.67,0.94$ & 0.69 & $0.58,0.83$ & 0.93 & $0.87,0.98$ \\
\hline$+\mathrm{SEP}$ & 2,951 & 1.00 & 0.81 & $0.69,0.96$ & 0.70 & $0.58,0.84$ & 0.92 & $0.87,0.98$ \\
\hline+ All confounders $* *$ & 2,951 & 1.00 & 0.82 & $0.69,0.97$ & 0.69 & $0.57,0.83$ & 0.92 & $0.86,0.98$ \\
\hline Test of proportional odds assumption & & & \multicolumn{4}{|c|}{$p=0.17$} & \multicolumn{2}{|c|}{$p=0.16$} \\
\hline Interaction: PA by gender & & & \multicolumn{4}{|c|}{$p=0.82$} & \multicolumn{2}{|c|}{$p=0.059$} \\
\hline Fully adjusted estimates using MICE to impute missing data & 3,298 & 1.00 & 0.82 & $0.70,0.96$ & 0.71 & $0.60,0.85$ & 0.90 & $0.85,0.96$ \\
\hline
\end{tabular}

MICE multiple imputation by chained equation

* OR derived from a proportional odds ordinal regression model adjusted for the time the accelerometer was worn

** Gender, age, markers of SEP (maternal education, housing tenure, household overcrowding, access to a car, highest parental social class), obesity (DXA fat mass adjusted for height) and substance use (binge drinking, cigarette smoking and cannabis use) 
MVPA and self-reported depressive symptoms at age 14 years

In univariable analyses, those who engaged in more MVPA had a reduced odds of depression (OR per $15 \mathrm{~min}$ : 0.87 (95\% CI: 0.82, 0.93)) (Table 1). This association attenuated following adjustment for gender, with the $95 \%$ confidence interval just excluding the null (OR per $15 \mathrm{~min}$ : 0.93 (95\% CI: 0.87, 0.99) (Table 1). Adjustment for additional confounders had little effect.

In the regression models described above, there was no evidence to suggest that the proportional odds assumption underpinning the ordinal regression models was not valid (Table 1). Similarly, there was no evidence for an interaction between gender and total PA (Table 1). There was weak evidence for an interaction between gender and MVPA (Table 1). Finally, there was no evidence of a nonlinear association between PA and depressive symptoms (data not shown).

Modelling total PA and percentage of time spent in MVPA to determine the importance of amount versus intensity of PA

Analyses were repeated to model the association between the percentage of time spent in MVPA (defined as tertiles) and tertiles of MFQ score (Table 2). Total PA (tertiles) and percentage of time spent in MVPA (tertiles) were then included in the same regression model to determine the relevance of the amount versus the intensity of the activity (Table 2). Increasing the total amount of PA undertaken was associated with a reduced odds of being depressed. However, increasing the percentage of time spent in MVPA was not independently associated with a reduction in the odds of being depressed. There was no evidence of collinearity between the two measures of physical activity (variance inflation factor $=1.4-1.7$ ).

Possible confounding by pubertal stage

Restricting the dataset to girls and adjusting for the timing of menarche had little effect on the effect estimates observed (Web Appendix 2). Thus, there was no evidence to suggest that pubertal stage confounded the observed association between higher levels of total PA and depressive symptoms amongst adolescent girls.

\section{Missing data}

Of the 5,423 clinic attenders, girls and those of higher SEP (based on maternal education) were more likely to wear the accelerometer (data not shown). Eighty-nine percent of those with data on physical activity and depressive symptoms ( $n=3,298$ ) were included in the main dataset for analysis. The remainder had missing data on one or more potential confounders. Imputation of missing data suggested that those adolescents whose data were imputed were more likely to be of lower SEP (Web Appendix 3).

Analyses including imputed data also found that adolescents who were more active (both in terms of total PA and minutes of MVPA) had reduced odds of depressive symptoms (Table 1). Subsequent models included both total PA and percentage of time in MVPA as tertiles. Effect estimates for analyses related to the percentage of time spent in MVPA were slightly attenuated compared to complete-case analyses. However, results supported the earlier findings that it was the total amount of PA undertaken that was associated with reduced odds of adolescent depression, rather than time spent in MVPA, once both were mutually adjusted by inclusion in the same model (Table 2). There was some evidence that those in the highest tertile of MVPA had a reduced odds of depression (OR imputed 0.86 (95\% CI: $0.73,1.01)$, but the $95 \%$ confidence interval just included the null and there was no evidence of a dose-response effect across tertiles.

\section{Discussion}

\section{Summary of findings}

In these cross-sectional analyses, adolescents who were more active had reduced odds of depressive symptoms. There was no evidence for a differential effect of PA according to gender. The intensity of the PA (i.e. percentage of time spent in MVPA) was not associated with depression in this cohort once total PA was taken into account. Thus, our cross-sectional results suggest that it is the amount of PA undertaken that is inversely associated with depressive symptoms in adolescents, rather than the intensity of that PA.

Strengths and limitations

The major strength of the ALSPAC cohort is its large size and the detailed information that has been collected. This has enabled adjustment for a wide range of potential confounders in the present analyses. The availability of objective data on PA recorded using accelerometry is a key feature of this cohort. This has enabled examination of the importance of the intensity of the PA.

The adolescents reported their mood using the MFQ. This widely used instrument has high reliability and validity [2]. Data on self-reported scores were used as it is known that parents are likely to underreport depressive symptoms in their child [9]. 


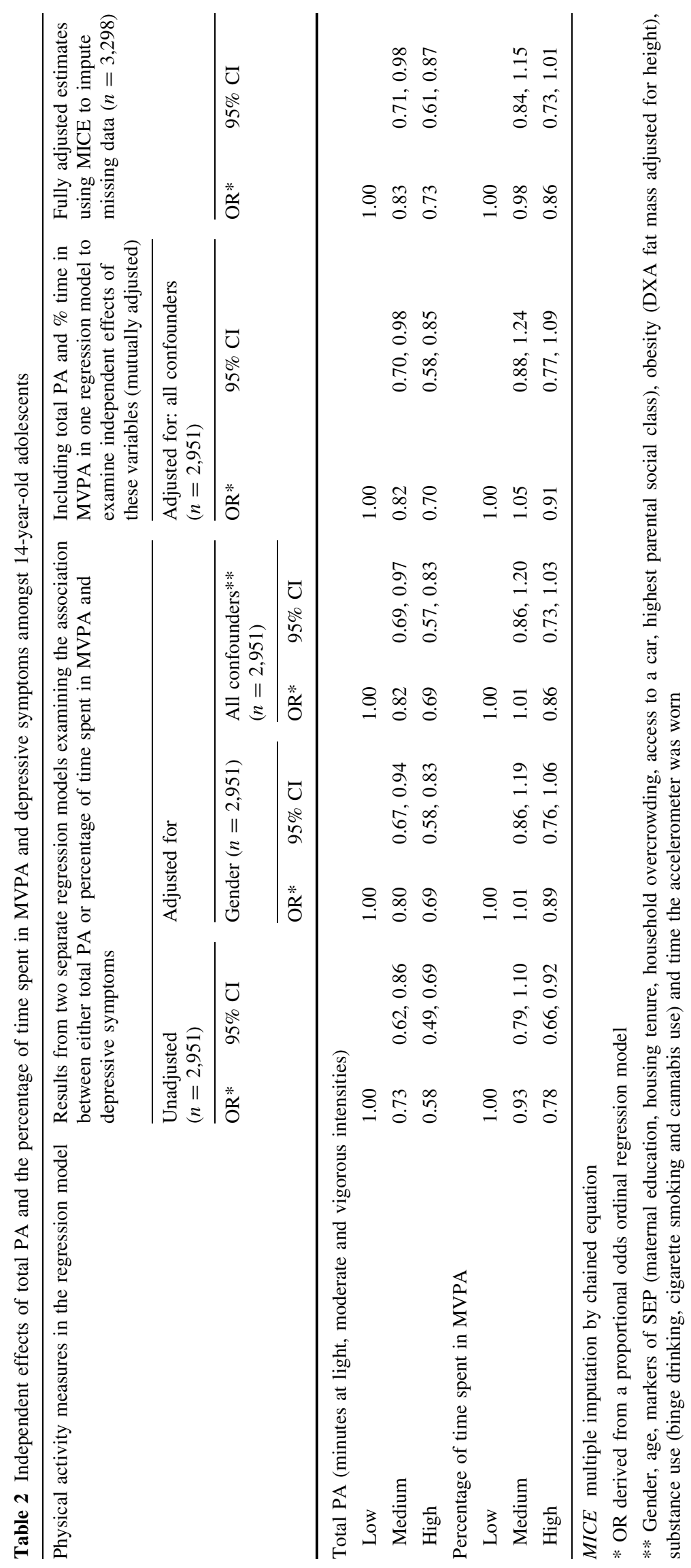


Although we have been able to adjust for a wide range of potential confounders, given the observational nature of the data, it is not possible to rule out the possibility of residual confounding. However, with the exception of gender, adjustment for confounders made little difference to the magnitude of the association.

Given that the present analyses are based on cross-sectional data, the temporal nature of the association cannot be elucidated. Thus, it may be that those adolescents who are depressed are less likely to engage in any activity. Moreover, it may be that participation in activities at higher intensities is particularly difficult for those who are depressed. It is also plausible that the association between PA and depression may be bi-directional. Large longitudinal studies with objective data on PA and repeated measures of adolescent depression, together with detailed information on potential confounders are therefore required to examine the temporal nature of this association.

Whilst the ALSPAC cohort is well characterised, $11 \%$ of those with data on PA and mood were excluded from the complete-case analyses due to missing data on one or more confounders. Such missingness not only has the potential to make effect estimates less precise, but may also introduce bias. Therefore, we conducted sensitivity analyses to examine the impact of missing data on our findings. The imputation model included a comprehensive list of variables, thus making the missing at random assumption that underpins the method of MICE more realistic. Comparison of those with observed and imputed data suggested that missingness was, primarily, amongst those of lower socioeconomic position (SEP). Whilst there was weak evidence that those of lower SEP were more active, conclusions drawn from the analyses including imputed data did not differ substantially from those from the completecase analyses suggesting that the missing data had not biased the findings. In the analyses including imputed data, there was weak evidence that high levels of percentage of time in MVPA may be associated with reduced odds of depressive symptoms, but the $95 \%$ confidence spanned unity and there was no dose-response effect across tertiles of percentage of time in MVPA.

\section{Comparison with existing literature}

Our findings are consistent with the majority of the existing literature in supporting an association between more frequent (self-report) PA and lower levels of depression amongst adolescents [3, 14, 17, 19, 24, 32, 39, 41, 44, 53, 57, 60]. Importantly, such an association persisted following adjustment for a wide range of confounders in our study. Prior studies have been limited in this respect [1, 3, $53,56,60]$. There was no evidence to support a differential effect of total PA on depression by gender, and only weak evidence for a differential effect of MVPA on depression by gender. The only other study that formally tested for an interaction found no evidence to support an interaction $(p>0.05)[1]$.

Only three prior studies have measured PA objectively. One used pedometers [42], which do not measure the intensity of the PA, and two used accelerometers [21, 43], as in the current study. Analyses of baseline data from the TAAG trial [21], which was designed to evaluate the effectiveness of an intervention to reduce the decline in PA in adolescent girls $(N=1,397)$, found no evidence to support an association between light, moderate or vigorous PA and depressive symptoms. An earlier small study of 57 primary school children aged 9-10 years found no evidence of association between overall PA and depressive symptoms ( $r=-0.014)$. However, increased vigorous PA was associated with a reduction in depressive symptoms $(r=-0.18)$ and, conversely, undertaking more activity at a very light intensity was associated with an increase in depressive symptoms $(r=0.35)$ [43]. In contrast, the results from the present study undertaken in a large representative UK population-based cohort of 14 year olds suggest that it was the amount of PA undertaken that was inversely associated with mental health in adolescents rather than the intensity of that activity.

Earlier analyses of data from the ALSPAC cohort found that time spent in MVPA was more important than total activity in terms of reducing obesity [37, 47]. Thus, the relevance of the intensity of the activity appears to be dependent on the health outcome examined. Whilst the cross-sectional nature of our study means that we cannot exclude the possibility of reverse causality (i.e. those who are depressed are less likely to be active), the findings suggest that different dimensions of PA may be important for different dimensions of health. If this is confirmed in longitudinal studies and, subsequently, in randomised controlled trials, this would have important implications for public health messages.

\section{Mechanisms}

Little is known about the potential mechanisms that may underlie an association between PA and mood. Animal studies have shown that PA increases levels of brainderived neurotrophic factor [63], promotes cell proliferation [7] and 5-hydroxytryptamine release [33], and others have shown that PA may reduce cortisol levels [36]. Furthermore, there is evidence that self-esteem [12] and/ or social support [50] may be important mediators. Alternatively, as others have suggested [22], physical activity may distract individuals from their negative thoughts or ruminative style [38, 48], which are key in depression. 
Given that it was the total amount of PA that was associated with reduced odds of depressive symptoms rather than the intensity of the activity, the context in which this PA is accumulated may be relevant. Adolescents who engage in activities (of a formal or informal nature) may increase their social networks, thus increasing their social support which, in turn, may protect against depression. The surrounding environment may also be important. Recent reports suggest that undertaking "green exercise" (activity in green areas) may also be beneficial [5]. Greater understanding of the factors that mediate the association between PA and health (both in terms of physical and mental health) will be key in ensuring clarity in public health messages.

\section{Conclusions and directions for further research}

In these cross-sectional analyses, adolescents who were more physically active (based on a measure of total PA) had reduced odds of depressive symptoms. Time spent in MVPA was not independently associated with depressive symptoms in this cohort. This suggests that it is the amount of PA undertaken that is important rather than the intensity of that activity for adolescent depressive symptoms. If confirmed in longitudinal studies, and subsequent randomised controlled trials, this may have important implications in terms of public health messages. It is important to promote both physical and mental health, but it may be that a different message is needed for each outcome. Research that helps elucidate the mechanism that underlies such associations will be important in helping to provide clarity for those promoting public health.

\begin{abstract}
Acknowledgments We are extremely grateful to all the families who took part in this study, the midwives for their help in recruiting them, and the whole ALSPAC team, which includes interviewers, computer and laboratory technicians, clerical workers, research scientists, volunteers, managers, receptionists and nurses. The UK Medical Research Council (Grant ref: 74882), the Wellcome Trust (Grant ref: 076467) and the University of Bristol provide core support for ALSPAC. DAL works in a centre that receives funding from the UK Medical Research Council (G0600705) and the University of Bristol. The funders have had no role in the writing of this report.
\end{abstract}

Open Access This article is distributed under the terms of the Creative Commons Attribution Noncommercial License which permits any noncommercial use, distribution, and reproduction in any medium, provided the original author(s) and source are credited.

\section{References}

1. Allison KR, Adlaf EM, Irving HM, Hatch JL, Smith TF, Dwyer JJ, Goodman J (2005) Relationship of vigorous physical activity to psychologic distress among adolescents. J Adolesc Health 37:164-166
2. Angold A, Costello EJ, Messer SC, Pickles A, Winder F, Silver D (1995) Development of a short questionnaire for use in epidemiological studies of depression in children and adolescents. Int $\mathbf{J}$ Methods Psychiatr Res 5:237-245

3. Babiss LA, Gangwisch JE (2009) Sports participation as a protective factor against depression and suicidal ideation in adolescents as mediated by self-esteem and social support. J Dev Behav Pediatr 30:376-384

4. Baranowski T, Dworkin RJ, Cieslik CJ (1984) Reliability and validity of self report of aerobic activity. Fam Health Proj Res Quart 55:309-317

5. Barton J, Pretty J (2010) What is the best dose of nature and green exercise for improving mental health? A multi-study analysis. Environ Sci Technol 44:3947-3955

6. Biddle SJH, Cavill N, Sallis J (1998) Policy framework for young people and health-enhancing physical activity. In: Biddle SJH, Sallis J, Cavill N (eds) Young and active? Young people and health-enhancing physical activity: evidence and implications. Health Education Authority, London, pp 3-16

7. Bjornebekk A, Mathe A, Brene S (2005) The antidepressant effect of running is associated with increased hippocampal cell proliferation. Int J Neuropsychopharmacol 8:357-368

8. Boreham C, Riddoch C (2001) The physical activity, fitness and health of children. J Sports Sci 19:915-929

9. Cole DA, Tram JM, Martin JM, Hoffman KB, Ruiz MD, Jacquez FM, Maschman TL (2002) Individual differences in the emergence of depressive symptoms in children and adolescents: a longitudinal investigation of parent and child reports. J Abnorm Psychol 111:156-165

10. Department of Health (2004) At least five a week. Evidence on the impact of physical activity and its relationship to health. Department of Health, London

11. Donovan JE (2009) Estimated blood alcohol concentrations for child and adolescent drinking and their implications for screening instruments. Pediatrics 123:e975-e981

12. Ekeland E, Heian F, Hagen KB (2005) Can exercise improve self esteem in children and young people? A systematic review of randomised controlled trials. Br J Sports Med 39:792-798

13. Ekelund U, Sjostrom M, Yngve A, Poortvliet E, Nilsson A, Froberg K, Wedderkopp N, Westerterp K (2001) Physical activity assessed by activity monitor and doubly labeled water in children. Med Sci Sports Exerc 33:275-281

14. Field T, Diego M, Sanders CE (2001) Exercise is positively related to adolescents' relationships and academics. Adolescence 36:105-110

15. Fulkerson JA, Sherwood NE, Perry CL, Neumark-Sztainer D, Story M (2004) Depressive symptoms and adolescent eating and health behaviors: a multifaceted view in a population-based sample. Prev Med 38:865-875

16. Golding J, Pembrey M, Jones R, ALSPAC Study Team (2001) ALSPAC-the Avon Longitudinal Study of Parents and Children. I. Study methodology. Paediatr Perinat Epidemiol 15:74-87

17. Haarasilta LM, Marttunen MJ, Kaprio JA, Aro HM (2004) Correlates of depression in a representative nationwide sample of adolescents (15-19 years) and young adults (20-24 years). Eur J Pub Health 14:280-285

18. Harro M, Riddoch C (2000) Physical activity. In: Armstrong N, van Mechelen W (eds) Paediatric exercise science and medicine. Oxford University Press, Oxford, pp 77-84

19. Hong X, Li J, Xu F, Ah Tse L, Liang Y, Wang Z, Tak-sun Yu I, Griffiths S (2009) Physical activity inversely associated with the presence of depression among urban adolescents in regional China. BMC Public Health 9:148. doi:10.1186/1471-2458-9148

20. Janssen I, LeBlanc AG (2010) Systematic review of the health benefits of physical activity and fitness in school-aged children 
and youth. Int J Behav Nutr Phys Act 7:40. doi:10.1186/14795868-7-40

21. Johnson CC, Murray DM, Elder JP, Jobe JB, Dunn AL, Kubik M, Voorhees C, Schachter K (2008) Depressive symptoms and physical activity in adolescent girls. Med Sci Sports Exerc 40:818-826

22. Just N, Alloy LB (1997) The response styles theory of depression: tests and an extension of the theory. J Abnorm Psychol 106:221-229

23. Kesaniemi YK, Danforth E Jr, Jensen MD, Kopelman PG, Lefebvre P, Reeder BA (2001) Dose-response issues concerning physical activity and health: an evidence-based symposium. Med Sci Sports Exerc 33:S351-S358

24. Kirkcaldy BD, Shephard RJ, Siefen RG (2002) The relationship between physical activity and self-image and problem behaviour among adolescents. Soc Psychiatry Psychiatr Epidemiol 37:544-550

25. Klerman GL, Weissman MM (1989) Increasing rates of depression. JAMA 261:2229-2235

26. Lewinsohn PM, Roberts RE, Seeley JR, Rohde P, Gotlib IH, Hops H (1994) Adolescent psychopathology: II: psychosocial risk factors for depression. J Abnorm Psychol 103:302-315

27. Lewinsohn PM, Rohde P, Klein DN, Seeley JR (1999) Natural course of adolescent major depressive disorder: I. Continuity into young adulthood. J Am Acad Child Adolesc Psychiatry 38:56-63

28. Mathers CD, Loncar D (2005) Updated projections of global mortality and burden of disease, 2002-2030: data sources, methods and results (working paper). WHO, Geneva

29. Mattocks C, Leary SD, Ness AR, Deere K, Saunders J, Tilling K, Kirkby J, Blair SN, Riddoch C (2007) Calibration of an accelerometer during free-living activities in children. Int $\mathrm{J}$ Pediatr Obes 2:218-226

30. Mattocks C, Ness AR, Deere K, Tilling K, Leary SD, Blair SN, Riddoch C (2008) Early life determinants of physical activity in 11-12 year olds: cohort study. BMJ 336:26-29

31. Mattocks C, Ness AR, Leary SD, Tilling K, Blair SN, Shield J, Deere K, Saunders J, Kirkby J, Davey Smith G, Wells J, Wareham N, Reilly J, Riddoch C (2008) Use of accelerometers in a large field-based study of children: protocols, design issues and effects on precision. J Phys Act Health 5:S98-S111

32. McDermott RJ, Hawkins WE, Marty PJ, Littlefield EA, Murray S, Williams TK (1990) Health behavior correlates of depression in a sample of high school students. J Sch Health 60:414417

33. Meeusen R, Thorre K, Chaouloff F, Sarre S, De Meirleir K, Ebinger G, Michotte Y (1996) Effects of trytophan and/or acute running on extracellular 5-HT and 5-HIAA levels in the hippocampus of food-deprived rats. Brain Res 740:245-252

34. Messer SC, Angold A, Costello EJ, Loeber R, Van Kammen W, Stouthamer-Loeber M (1995) Development of a short questionnaire for use in epidemiological studies of depression in children and adolescents: factor composition and structure across development. Int J Methods Psychiatr Res 5:251-262

35. Milligan RA, Burke V, Beilin LJ, Richards J, Dunbar D, Spencer M, Balde E, Gracey MP (1997) Health-related behaviours and psycho-social characteristics of 18 year-old Australians. Soc Sci Med 45:1549-1562

36. Nabkasorn C, Miyai N, Sootmongkol A, Junprasert S, Yamamoto H, Arita M, Miyashita K (2006) Effects of physical exercise on depression, neuroendocrine stress hormones and physiological fitness in adolescent females with depressive symptoms. Eur J Pub Health 16(2):179-184

37. Ness AR, Leary SD, Mattocks C, Blair SN, Reilly JJ, Wells J, Ingle S, Tilling K, Davey Smith G, Riddoch C (2007) Objectively measured physical activity and fat mass in a large cohort of children. PLoS Med 4:e97. doi:10.1371/journal.pmed.0040097
38. Nolen-Hoeksema S (1991) Responses to depression and their effects on the duration of depressive episodes. J Abnorm Psychol 100:569-582

39. Norris R, Carroll D, Cochrane R (1992) The effects of physical activity and exercise training on psychological stress and wellbeing in an adolescent population. J Psychosom Res 36:55-65

40. Office of Population Censuses and Surveys (1991) Standard Occupational Classification. HMSO, London

41. Page RM, Tucker LA (1994) Psychosocial discomfort and exercise frequency: an epidemiological study of adolescents. Adolescence 29:183-191

42. Parfitt G, Eston RG (2005) The relationship between children's habitual activity level and psychological well-being. Acta Paediatr 94:1791-1797

43. Parfitt G, Pavey T, Rowlands AV (2009) Children's physical activity and psychological health: the relevance of intensity. Acta Paediatr 98:1037-1043

44. Pastor Y, Balaguer I, Pons D, Garcia-Merita M (2003) Testing direct and indirect effects of sports participation on perceived health in Spanish adolescents between 15 and 18 years of age. J Adolesc 26:717-730

45. Perera B, Torabi MR, Jayawardana G, Pallethanna N (2006) Depressive symptoms among adolescents in Sri Lanka: prevalence and behavioral correlates. J Adolesc Health 39:144-146

46. Riddoch C (1998) Relationships between physical activity and physical health in young people. In: Biddle SJH, Sallis J, Cavill N (eds) Young and active? Young people and health-enhancing physical activity: evidence and implications. Health Education Authority, London, pp 17-48

47. Riddoch CJ, Leary SD, Ness AR, Blair SN, Deere K, Mattocks C, Griffiths A, Davey Smith G, Tilling K (2009) Prospective associations between objective measures of physical activity and fat mass in 12-14 year old children: the Avon Longitudinal Study of Parents and Children (ALSPAC). BMJ 339:b4544. doi: 10.1136/bmj.b4544

48. Rood L, Roelofs J, Bogels SM, Nolen-Hoeksema S, Schouten E (2009) The influence of emotion-focused rumination and distraction on depressive symptoms in non-clinical youth: a metaanalytic review. Clin Psychol Rev 29:607-616

49. Sallis JF (1991) Self-report measures of children's physical activity. J Sch Health 61:215-219

50. Seefeldt V, Malina RM, Clark MA (2002) Factors affecting levels of physical activity in adults. Sports Med 32:143-168

51. Sirard JR, Pate RR (2001) Physical activity assessment in children and adolescents. Sports Med 31:439-454

52. Stata Corp (2008) Stata Statistical Software: Release 10.1. College Station, TX: Stata Corporation

53. Steptoe A, Butler N (1996) Sports participation and emotional wellbeing in adolescents. Lancet 347:1789-1792

54. Strong WB, Malina RM, Blimkie CJ, Daniels SR, Dishman RK, Gutin B, Hergenroeder AC, Must A, Nixon PA, Pivarnik JM, Rowland T, Trost S, Trudeau F (2005) Evidence based physical activity for school-age youth. J Pediatr 146:732-737

55. Sund AM, Larsson B, Wichstrom L (2011) Role of physical and sedentary activities in the development of depressive symptoms in adolescence. Soc Psychiatry Psychiatr Epidemiol 46:431-441

56. Tao FB, Xu ML, Sun Y, Su PY, Huang K (2007) Physical activity might not be the protective factor for health risk behaviours and psychopathological symptoms in adolescents. J Paediatr Child Health 43:762-767

57. Trainor S, Delfabbro P, Anderson S, Winefield A (2010) Leisure activities and adolescent psychological well-being. J Adolesc 33:173-186

58. Trost SG, Pate RR, Freedson PS, Sallis JF, Taylor WC (2000) Using objective physical activity measures with youth: how many 
days of monitoring are needed? Med Sci Sports Exerc $32: 426-431$

59. Twisk JW (2001) Physical activity guidelines for children and adolescents: a critical review. Sports Med 31:617-627

60. Ussher MH, Owen CG, Cook DG, Whincup PH (2007) The relationship between physical activity, sedentary behaviour and psychological wellbeing among adolescents. Soc Psychiatry Psychiatr Epidemiol 42:851-856

61. van Buuren S, Boshuizen HC, Knook DL (1999) Multiple imputation of missing blood pressure covariates in survival analysis. Stat Med 18:681-694
62. Wareham NJ, Rennie KL (1998) The assessment of physical activity in individuals and populations: why try to be more precise about how physical activity is assessed? International Journal of Obesity \& Related Metabolic Disorders: Journal of the International Association for the Study of Obesity Suppl 2:S30-S38

63. Zheng H, Liu Y, Li W, Yang B, Chen D, Wang X, Jiang Z, Wang H, Wang Z, Cornelisson G, Halberg F (2006) Beneficial effects of exercise and its molecular mechanisms on depression in rats. Behav Brain Res 168:47-55 